NIKHEF / 2004-016

ITP-UU-04/41

Cavendish-HEP-2004/31

DAMTP-2004-130

hep-ph/0411241

\title{
Joint resummation for heavy quark production
}

\author{
Andrea BANFI $^{a, b, c}$, ERIC LAENEN ${ }^{a, d}$ \\ ${ }^{a}$ NIKHEF Theory Group \\ Kruislaan 409, 1098 SJ Amsterdam, The Netherlands \\ ${ }^{b}$ Cavendish Laboratory, University of Cambridge \\ Madingley Road, CBз OHE Cambridge, UK \\ ${ }^{c}$ DAMTP, Centre for Mathematical Sciences \\ Wilberforce Road, CB3 0WA Cambridge, UK \\ ${ }^{d}$ Institute for Theoretical Physics, Utrecht University \\ Leuvenlaan 4, 3584 CE Utrecht, The Netherlands
}

\begin{abstract}
We present joint threshold and recoil resummed transverse momentum distributions for heavy quark hadroproduction, at next-to-leading logarithmic accuracy. We study the dependence of these distributions on the production channel, the color configurations and the differences with the pure threshold-resummed distribution.
\end{abstract}




\section{Introduction}

The formalism [1, 2] of hadronic cross sections for the joint resummation of distributions singular at partonic threshold and at zero recoil has so far been applied to only a few processes. The most recent studies involve processes that proceed at lowest order through a $2 \rightarrow 1$ electroweak ( $Z / W$ production [3]) or Yukawa interaction (Higgs production [4]). For these cases, the observables are the production cross sections at fixed mass $Q$ and measured $Q_{T}$. Partonic threshold is defined by $z \equiv Q^{2} / \hat{s}=1$, where $\hat{s}$ is the partonic center of mass energy squared, and zero recoil by $Q_{T}=0$. At any finite order, the distributions take the form of plus-distributions $\left[\ln ^{k}(1-z) /(1-z)\right]_{+}$and $\left[\ln ^{k}\left(Q / Q_{T}\right) / Q_{T}\right]_{+}$. In these observables the latter distributions enter in the physical cross sections, whereas the former are defined, after factorization, in the context of a perturbative analysis of the hard scattering.

The basis for the joint threshold and recoil resummation for these processes and those proceeding at lowest order through a $2 \rightarrow 2$ (QCD) reaction was given in Ref. [2]. The first application of the formalism to the specific case of prompt photon hadroproduction was presented in Ref. [5]. There the photon transverse momentum spectrum was analyzed, and a preliminary numerical study performed. Recoil corrections were found to have a significant impact on the photon spectrum, both at large and at small values of $p_{T}$. For such $2 \rightarrow 2$ processes, the formalism implements the notion that, in the presence of QCD radiation, the actual transverse momentum produced by the hard collision is not $\vec{p}_{T}$ but rather $\vec{p}_{T}-\vec{Q}_{T} / 2$, with $\vec{Q}_{T}$ the total transverse momentum of soft recoiling partons. In fact, the joint-resummed partonic $p_{T}$ spectrum has the form of a hard scattering cross section as a function of $p_{T}^{\prime} \equiv\left|\vec{p}_{T}-\vec{Q}_{T} / 2\right|$, convoluted with a perturbative, albeit resummed $\vec{Q}_{T}$ distribution. The extreme situation $Q_{T}=2 p_{T}$ in which all transverse momentum is produced through recoil leads to a singularity in the hard scattering function, equivalent to the singularity at zero $p_{T}$ in the prompt-photon Born cross section. We note however that a recently proposed extension [6] of joint resummation avoids this singularity.

In this paper we apply the joint resummation formalism as given in Ref. [2] to another prominent $2 \rightarrow 2$ scattering observable: the $p_{T}$ distribution of heavy quarks produced in hadronic collisions. Key differences with the prompt-photon case are, first, the presence of the heavy quark mass $m$, preventing a singularity in the hard scattering function when $Q_{T}=2 p_{T}$ and, second, the possibility of multiple colored states for the produced top quark pair. We derive joint-resummed and threshold-resummed transverse momentum distributions at next-to-leading logarithmic (NLL) accuracy, and study for different production channels the dependence on color configurations, as well as differences among the two resummations and the exact next-to-leading order (NLO) distribution.

Other recent studies for heavy quark $p_{T}$ distributions based on finite order expansions of threshold resummation can be found in Refs. [7, 8].

Relevant formulas are derived in section 2. In section 3 we present numerical studies of the dependence of the joint resummation effects on the production channel, on color configurations and on the heavy quark flavor. We also assess the differences with the pure threshold-resummed distribution. Section 4 contains our conclusions. 


\section{The observable}

We consider the inclusive $p_{T}$ distribution of a heavy quark produced via the strong interaction in a hadron-hadron collision at center of mass $(\mathrm{cm})$ energy $\sqrt{S}$

$$
h_{A}\left(p_{A}\right)+h_{B}\left(p_{B}\right) \rightarrow Q\left(p_{c}\right)+X,
$$

where $h_{A, B}$ refers to the two incoming hadrons, $Q$ to the detected heavy quark and $X$ to the unobserved part of the final state which includes also the heavy anti-quark $\bar{Q}$. The lowest order QCD processes producing a heavy quark are

$$
\begin{aligned}
& q\left(p_{a}\right)+\bar{q}\left(p_{b}\right) \rightarrow Q\left(p_{c}\right)+\bar{Q}\left(p_{d}\right), \\
& g\left(p_{a}\right)+g\left(p_{b}\right) \rightarrow Q\left(p_{c}\right)+\bar{Q}\left(p_{d}\right),
\end{aligned}
$$

at cm energy $\sqrt{\hat{s}}=\sqrt{\xi_{a} \xi_{b} S}$ with $\xi_{a}, \xi_{b}$ parton momentum fractions. Exact higher order corrections to the differential cross sections for these partonic processes have been computed to NLO [9, 10, 11, 12. To any order [13] the observable may be written in the following factorized form (up to power corrections behaving as $1 / p_{T}^{2}$ )

$$
\frac{d \sigma_{A B \rightarrow Q+X}}{d p_{T}}=\sum_{a, b} \int_{0}^{1} d \xi_{a} d \xi_{b} \phi_{a / A}\left(\xi_{a}, \mu\right) \phi_{b / B}\left(\xi_{b}, \mu\right) \frac{d \hat{\sigma}_{a b \rightarrow Q+X}}{d p_{T}}\left(\xi_{a}, \xi_{b}, \alpha_{s}(\mu), p_{T}\right),
$$

with $d \hat{\sigma}_{a b \rightarrow Q+X} / d p_{T}$ the partonic differential cross-section, $\phi_{a / A}$ and $\phi_{b / B}$ parton densities, and $\mu$ the factorization and renormalization scale. The purpose of this study is to investigate certain effects of soft gluons in these higher order corrections for the heavy quark $p_{T}$ distribution.

The first of these are threshold enhancements, which essentially involve the energy of soft gluons. In the context of the factorization (3) we define hadronic and partonic threshold by the conditions $S=4 m_{T}^{2}$ and $\hat{s}=4 m_{T}^{2}$, respectively, with $m_{T}$ the transverse mass $\sqrt{m^{2}+p_{T}^{2}}$. It is convenient to define the scaling variables

$$
x_{T}^{2}=\frac{4 m_{T}^{2}}{S}, \quad \hat{x}_{T}^{2}=\frac{4 m_{T}^{2}}{\xi_{a} \xi_{b} S},
$$

so that hadronic (partonic) threshold is at $x_{T}^{2}=1\left(\hat{x}_{T}^{2}=1\right)$. The higher order corrections to the partonic cross section $d \hat{\sigma}_{a b} / d p_{T}$ contain distributions that are singular at partonic threshold. Threshold resummation organizes such distributions to all orders.

The second of these are recoil effects, resulting from radiation of soft gluons from initial-state partons. We wish to treat these effects in the context of joint threshold and recoil resummation. To this end we employ the refactorization analysis of Refs. [2, 5], which enables us to identify a hard scattering with reduced $\mathrm{cm}$ energy squared $Q^{2}$ and at transverse momentum $\vec{Q}_{T}$ with respect to the hadronic cm system. This hard scattering produces a heavy quark with transverse momentum

$$
\vec{p}_{T}^{\prime} \equiv \vec{p}_{T}-\frac{\vec{Q}_{T}}{2}
$$

The kinematically allowed range for the invariant mass $Q$ of the heavy quark pair in this hard scattering is limited from below by $2 m_{T}^{\prime}=2 \sqrt{m^{2}+p_{T}^{\prime 2}}$ so that threshold in the context of joint resummation is defined by

$$
\tilde{x}_{T}^{2} \equiv \frac{4 m_{T}^{\prime 2}}{Q^{2}}=1
$$


A refactorization analysis on the lines of the one performed in Ref. [2] leads to the following expression for the observable in Eq. (3)

$$
\frac{d \sigma_{A B \rightarrow Q+X}}{d p_{T}}=\int d^{2} Q_{T} d Q^{2} \theta\left(\bar{\mu}-\left|\vec{Q}_{T}\right|\right) \frac{d \sigma_{A B \rightarrow Q+X}}{d p_{T} d Q^{2} d^{2} \vec{Q}_{T}}
$$

where

$$
\begin{aligned}
\frac{d \sigma_{A B \rightarrow Q}+X}{d p_{T} d Q^{2} d^{2} \vec{Q}_{T}}=\sum_{a b=q \bar{q}, g g} \int d \xi_{a} d \xi_{b} \phi_{a / A}\left(\xi_{a}, \mu\right) \phi_{b / B}\left(\xi_{b}, \mu\right) \\
\quad \times \int_{0}^{\xi_{a}} d x_{a} d^{2} k_{a} \bar{c}_{a / a}\left(\frac{x_{a}}{\xi_{a}}, \vec{k}_{a}\right) \int_{0}^{\xi_{b}} d x_{b} d^{2} k_{b} \bar{c}_{b / b}\left(\frac{x_{b}}{\xi_{b}}, \vec{k}_{b}\right) \\
\quad \times \frac{d p_{T}^{\prime}}{d p_{T}} \int_{0}^{1} d w_{s}\left\{C_{\delta, \mathbf{1}}^{a b}\left(\alpha_{s}(\mu), \tilde{x}_{T}^{2}\right) \frac{d \sigma_{a b, \mathbf{1}}^{(0)}}{d p_{T}^{\prime}} S_{\mathbf{1}}^{\prime}\left(w_{s}\right)+C_{\delta, \mathbf{8}}^{a b}\left(\alpha_{s}(\mu), \tilde{x}_{T}^{2}\right) \frac{d \sigma_{a b, \mathbf{8}}^{(0)}}{d p_{T}^{\prime}} S_{\mathbf{8}}^{\prime}\left(w_{s}\right)\right\} \\
\quad \times \frac{1}{S} \delta\left(1-Q^{2} / S-\left(1-x_{a}\right)-\left(1-x_{b}\right)-w_{s}\right) \delta\left(\vec{Q}_{T}+\vec{k}_{a}+\vec{k}_{b}\right)
\end{aligned}
$$

is known as the profile function. Note that we restrict ourselves to relatively soft, perturbative recoil contributions by integrating over $\vec{Q}_{T}$ in Eq. (7) such that $\left|\vec{Q}_{T}\right| \leq \bar{\mu}$. Implementing a cut-off $\bar{\mu}$ is of course a rather crude approximation to what should be a consistent matching to finite order results. The implementation of such a matching procedure is however beyond the scope of this work. ${ }^{1}$ We shall discuss the limitations on the integration variables such as $\vec{Q}_{T}$ further in the next section.

Let us comment on the right hand side of Eq. (8), moving from bottom to top. The last line implements transverse momentum conservation, as well as the decomposition of the (normalized) above-threshold energy $1-Q^{2} / S$ into contributions from collinear radiation $\left(1-x_{a, b}\right)$ and from wide-angle soft radiation $\left(w_{s}\right)$. The definition of the observable and the refactorization ensure [2] that only the initial state contributes to the recoil of the hard scattering. The line above the last displays the hard scattering functions $C_{\delta} d \sigma^{(0)} / d p_{T}^{\prime}$, one per color state (singlet or octet) in which the heavy quark pair can be produced. In a diagrammatic representation all its lines are off-shell by at least an amount $p_{T}^{\prime}$. The factor $d p_{T}^{\prime} / d p_{T}$ accounts for the phase space difference between the hard scattering in the factorized expression Eq. (3) $\left(d \sigma^{(0)} / d p_{T}\right)$ and here, where $d \sigma^{(0)} / d p_{T}^{\prime}$ is appropriate. The functions $S_{\mathbf{1}}^{\prime}$ and $S_{\mathbf{8}}^{\prime}$ summarize the effects of coherent wide-angle soft radiation, and are sensitive to the color structure of the hard scattering. In particular, the effect of soft radiation for our observable is such that, to NLL accuracy, there is no mixing between singlet and octet color states, as was already observed for the case of the total heavy-quark cross section near threshold [14].

Moving up further in Eq. (8), the functions $\bar{c}_{a / a}$ are defined in Ref. [2], and are related to the density of parton $a$ in a parton of the same flavor at fixed energy fraction $x_{a}$ and transverse momentum $\vec{k}_{a}$. The prime on the $S$ function indicates that factors $\sqrt{U_{D Y}}$, the square root of the Drell-Yan soft function, have been introduced in equal numbers in numerator and denominator and then conveniently redistributed between the heavy quark soft functions and the $\bar{c}$ functions in order to remove gauge dependence in the individual functions. Finally the top line displays the convolution with standard parton distribution

\footnotetext{
${ }^{1}$ Note that such a matching in the extension of Ref. [6] is straightforward.
} 
functions, as well as the sum over initial state parton flavors, in which the $g q, g \bar{q}$ channels have been neglected because they are suppressed near threshold.

A convenient way to implement the energy and transverse momentum conservation is by passing to transform space, where the impact vector $\vec{b}$ is Fourier conjugate to $\vec{Q}_{T}$, and the variable $N$ is Mellin conjugate to the threshold variables in Eqns. (41) and (6). This converts the convolution in Eq. (8) to a product. We aim at NLL accuracy in transform space, that is we account for all contributions $\exp \left\{\alpha_{s}^{n} L^{n+1}\right\}$ and $\exp \left\{\alpha_{s}^{n} L^{n}\right\}$, where $L$ can be either $\ln N$ or $\ln b$.

Using their renormalization group scaling properties, moments of the soft functions may, to next-to-leading logarithmic (NLL) accuracy, be written as [15, 16]

$$
\tilde{S}_{\mathbf{1} / \mathbf{8}}^{\prime}\left(\frac{Q}{N \mu}, \alpha_{s}(\mu)\right)=\tilde{S}_{\mathbf{1} / \mathbf{8}}^{\prime}\left(1, \alpha_{s}(Q / N)\right) \exp \left[\int_{\mu}^{Q / N} \frac{d \mu^{\prime}}{\mu^{\prime}} 2 \operatorname{Re} \Gamma_{\mathbf{1} / \mathbf{8}}\left(\alpha_{s}\left(\mu^{\prime}\right)\right)\right] .
$$

The solution needs careful definition in order to avoid values of $N$ for which $\alpha_{s}(Q / N)$ is singular. The $N$-integration contour we choose indeed assures this, and anyhow, at NLL accuracy, we can approximate the functions $\tilde{S}_{\mathbf{1 / 8}}^{\prime}\left(1, \alpha_{s}\right)$ by their lowest order expressions. The form of the one-loop soft anomalous dimensions $\Gamma_{1}$ and $\Gamma_{\mathbf{8}}$ we need is straightforwardly derived from the expressions in Ref. [15, 16, 17]. Their real parts are

$$
\begin{aligned}
& 2 \operatorname{Re} \Gamma_{\mathbf{1}}\left(\alpha_{s}\right)=-\frac{\alpha_{s}}{\pi} 2 C_{F}\left(\operatorname{Re} L_{\beta}+1\right), \\
& 2 \operatorname{Re} \Gamma_{\mathbf{8}}\left(\alpha_{s}\right)=2 \operatorname{Re} \Gamma_{\mathbf{1}}\left(\alpha_{s}\right)+\frac{\alpha_{s}}{\pi} C_{A}\left(\ln \frac{m_{T}^{2}}{m^{2}}+\operatorname{Re} L_{\beta}\right), \\
& \operatorname{Re} L_{\beta}=\frac{1+\beta^{2}}{2 \beta}\left(\ln \frac{1-\beta}{1+\beta}\right), \quad \beta=\sqrt{1-m^{2} / m_{T}^{2}} .
\end{aligned}
$$

We postpone a discussion of these expressions to further below.

To NLL accuracy we may approximate both $d p_{T}^{\prime} / d p_{T}$ and the $C_{\delta}$ functions by 1 , so that integrating over $Q^{2}$, and, with manipulations similar to those of Ref. [2] we arrive at

$$
\begin{aligned}
& \frac{d \sigma_{A B \rightarrow Q+X}}{d p_{T} d^{2} \vec{Q}_{T}}=\sum_{a b=q \bar{q}, g g} p_{T} \int \frac{d^{2} b}{(2 \pi)^{2}} e^{i \vec{b} \cdot \vec{Q}_{T}} \int \frac{d N}{2 \pi i} \phi_{a / A}(N, \mu) \phi_{b / B}(N, \mu) e^{E_{a b}(N, b)} \\
& \frac{e^{-2 C_{F} t(N)\left(\operatorname{Re} L_{\beta}+1\right)}}{4 \pi S^{2}}\left(\tilde{M}_{\mathbf{1}}^{2}(N)+\tilde{M}_{\mathbf{8}}^{2}(N) e^{C_{A} t(N)\left(\ln \frac{m_{T}^{2}}{m^{2}}+L_{\beta}\right)}\right)\left(\frac{S}{4\left(m^{2}+\left|\vec{p}_{T}-\vec{Q}_{T} / 2\right|^{2}\right)}\right)^{N+1} .
\end{aligned}
$$

where the inverse Fourier transform and Mellin transform are explicit. Notice in particular the last factor, which provides a kinematic link between recoil and threshold effects. The exponential functions $E_{a b}$ [2, 5] to next-to-leading logarithmic (NLL) accuracy are

$$
E_{a b}(N, b)=\int_{\chi(N, b)}^{Q} \frac{d \mu^{\prime}}{\mu^{\prime}}\left[A_{a}\left(\alpha_{s}\left(\mu^{\prime}\right)\right)+A_{b}\left(\alpha_{s}\left(\mu^{\prime}\right)\right)\right] 2 \ln \frac{\bar{N} \mu^{\prime}}{Q}-g b^{2}, \quad \bar{N}=N e^{\gamma_{E}}
$$

where the coefficients $A_{a}$ and $A_{b}$ are taken from Ref. [2. We also added to the perturbative exponent the non-perturbative (NP) Gaussian smearing term $-g b^{2}$. 
To simplify notations, we have combined in Eq. (13) $\tilde{S}_{1}^{\prime}, \tilde{S}_{\mathbf{8}}^{\prime}$ with the Born matrix elements that contribute to $d \sigma^{(0)} / d p_{T}$ to build up the two Born functions $\tilde{M}_{\mathbf{1}}^{2}(N)$ and $\tilde{M}_{\mathbf{8}}^{2}(N)$, and we have introduced the evolution variable

$$
t(N)=\int_{Q}^{Q / N} \frac{d \mu^{\prime}}{\mu^{\prime}} \frac{\alpha_{s}\left(\mu^{\prime}\right)}{\pi} .
$$

The Born functions $\tilde{M}_{\mathbf{1}}^{2}(N), \tilde{M}_{\mathbf{8}}^{2}(N)$ are the Mellin moments of the lowest order heavy quark production matrix elements for either the $q \bar{q}$ or $g g$ channel, the index labeling the color-state of the heavy quark pair:

$$
\begin{gathered}
\tilde{M}_{q \bar{q}, \mathbf{8}}^{2}(N) \equiv \int_{0}^{1} d \hat{x}_{T}^{2} \frac{\left(\hat{x}_{T}^{2}\right)^{N}}{\sqrt{1-\hat{x}_{T}^{2}}} M_{q \bar{q}, \mathbf{8}}^{2}\left(\hat{x}_{T}^{2}\right) \\
=16 \pi^{2} \alpha_{s}^{2} \frac{C_{F} T_{R}}{N_{c}} \frac{\sqrt{\pi} \Gamma(N+1)}{\Gamma(N+5 / 2)}\left(N+2+(N+1) \frac{m^{2}}{m_{T}^{2}}\right), \\
\tilde{M}_{g g, \mathbf{1}}^{2}(N) \equiv \int_{0}^{1} d \hat{x}_{T}^{2} \frac{\left(\hat{x}_{T}^{2}\right)^{N}}{\sqrt{1-\hat{x}_{T}^{2}}} M_{g g, \mathbf{1}}^{2}\left(\hat{x}_{T}^{2}\right) \\
=16 \pi^{2} \alpha_{s}^{2} \frac{2 T_{R}}{N_{c}\left(N_{c}^{2}-1\right)} \frac{\sqrt{\pi} \Gamma(N+1)}{\Gamma(N+3 / 2)}\left(\frac{N+1}{N}+2\left(\frac{m^{2}}{m_{T}^{2}}-\frac{m^{4}}{m_{T}^{4}}\right)\right), \\
\tilde{M}_{g g, \mathbf{8}}^{2}(N) \equiv \int_{0}^{1} d \hat{x}_{T}^{2} \frac{\left(\hat{x}_{T}^{2}\right)^{N}}{\sqrt{1-\hat{x}_{T}^{2}}} M_{g g, \mathbf{8}}^{2}\left(\hat{x}_{T}^{2}\right) \\
=\frac{16 \pi^{2} \alpha_{s}^{2}}{N_{c}^{2}-1} \frac{\sqrt{\pi} \Gamma(N+1)}{\Gamma(N+3 / 2)}\left[\left(2 C_{F}-\frac{1}{N_{c}}\right)\left(\frac{N+1}{N}+2\left(\frac{m^{2}}{m_{T}^{2}}-\frac{m^{4}}{m_{T}^{4}}\right)\right)\right. \\
\left.\quad-\frac{C_{A}}{2 N+3}\left(\frac{N+1}{N}+2(N+1)\left(\frac{m^{2}}{m_{T}^{2}}-\frac{m^{4}}{m_{T}^{4}}\right)\right)\right]
\end{gathered}
$$

with $M_{\mathbf{1}}^{2}$ and $M_{\mathbf{8}}^{2}$ the square matrix element for the production of a hard $Q \bar{Q}$ system in a singlet and octet color state respectively. We point out that, at this order, in the $q \bar{q}$ channel the heavy quark-antiquark pair is produced only in an octet state.

In a refactorization analysis, the expressions $2 \operatorname{Re} \Gamma_{\mathbf{1}}$ and $2 \mathrm{Re} \Gamma_{\mathbf{8}}$ are related to the (real parts of) anomalous dimensions of certain operators, composed of Wilson lines [16, 18, 19], as the structure of Eqs. (9) reflects. Because for our case imaginary parts of the virtual corrections (Coulomb phases) to the anomalous dimension cancel in the cross section, the soft function can be given a natural probabilistic interpretation.

From this viewpoint, at NLL accuracy, soft wide-angle gluons can be considered as being emitted independently from the external partons, and approaching threshold is equivalent to suppressing all soft gluon emissions with energies above $Q / N$. Furthermore, due to the fact that there is no mixing between different color structures, soft gluon emission exponentiates straightforwardly. The exponential in Eq. (91) can then be interpreted as the probability of not emitting any wide-angle soft gluon with energy above $Q / N$. We now offer some comments on the explicit expression of the anomalous dimensions in some limiting cases. 
- In the case in which the heavy quarks are at rest in their $\mathrm{cm}$ frame, i.e. $\beta \rightarrow 0$, $\operatorname{Re} L_{\beta} \rightarrow-1$, and $2 \operatorname{Re} \Gamma_{1}$ vanishes, corresponding to the fact that the combined heavy quark-anti-quark pair forms a color-singlet. On the other hand, $2 \operatorname{Re} \Gamma_{\mathbf{8}} \rightarrow-\alpha_{s} C_{A} / \pi$, which reflects the fact that the quark-antiquark pair produced in an octet state radiates as a single object rather than two separate particles.

- For positive moderate values of $\beta(0<\beta<1)$ we observe that $\Gamma_{\mathbf{8}}$ contains the term

$$
\ln \left(\frac{m_{T}^{2}}{m^{2}}\right)=\ln \left(\frac{\left(2 p_{a} \cdot p_{c}\right)\left(2 p_{b} \cdot p_{c}\right)}{\left(2 p_{a} \cdot p_{b}\right) m^{2}}\right),
$$

which is the standard contribution to the anomalous dimension from the $q \bar{q} g$ antenna.

- In the extreme region $p_{T} \gg m(\beta \rightarrow 1)$, gluons can become effectively collinear to the heavy quarks. In this case $L_{\beta}$ approximates $\ln p_{T}^{2} / m^{2}$, a large collinear logarithm. The resulting logarithmic contributions are identical in $\Gamma_{\mathbf{1}}$ and $\Gamma_{\mathbf{8}}$, reflecting the fact that collinear radiation depends only on the color charge of each hard emitting parton, and is not sensitive to the color or geometrical structure of the event. In this large $p_{T}$ limit, collinear logarithms should be resummed to all orders, and a different analysis is required [20, 21].

This completes our discussion of the joint-resummed transverse momentum distribution. The threshold-resummed result can now easily be derived, by substituting Eq. (13) into (7) (after the performing the $Q^{2}$ integral) and neglecting $\vec{Q}_{T}$ in the last factor in Eq. (13). Then the $\vec{Q}_{T}$ integral sets $\vec{b}$ to zero everywhere, yielding the threshold-resummed result.

\section{Top and bottom quark $p_{T}$ spectra}

In this section we demonstrate numerically the soft gluon effects for top and bottom quark transverse momentum distributions for collider and fixed target kinematics respectively. Rather than performing a detailed comparison with data, our aim is to examine the numerical behavior of our formulas in realistic settings. We leave therefore also to a future study the application of our results for the description of charm quark/charmed meson $p_{T}$ spectra in fixed target kinematics.

Let us first comment on the allowed integration range of the recoil momentum $Q_{T}$. In Eq. (7) we have simply imposed an upper limit $\bar{\mu}$ on the modulus of this transverse momentum vector. There is however another constraint on $Q_{T}$, because the recoil factor at the end of Eq. (113) implies the condition

$$
\left(\frac{S}{4\left(m^{2}+\left|\vec{p}_{T}-\vec{Q}_{T} / 2\right|^{2}\right)}\right)>1,
$$

which expresses the fact that the available hadronic energy be more than the minimum mass of the final state in the recoiling hard scattering. Clearly, for the maximum value

$p_{T}^{2}=S / 4-m^{2}, \vec{Q}_{T}$ has no phase space left. For a given value $p_{T}$ and (large) $\bar{\mu}$ the allowed range can be chosen to be the overlap of the two disks $D_{1,2}$ in $\vec{Q}_{T}$ space

$$
D_{1}: \quad\left|\vec{Q}_{T}-2 \vec{p}_{T}\right|^{2} \leq S-4 m^{2}, \quad D_{2}: \quad\left|\vec{Q}_{T}\right| \leq \bar{\mu} .
$$


or one can choose $\bar{\mu}$ such that disk $D_{2}$ fits in $D_{1}$. We chose the former option. Notice that due to the heavy quark mass, the expression in Eq. (13) stays finite at $Q_{T}=2 p_{T}$, in contrast to the prompt photon production case analyzed in Refs. 22, 5].

For top quark production we chose the cut-off $\bar{\mu}=200 \mathrm{GeV}$. This might seem quite large as a cut-off on recoil momentum, given that the joint-resummation formalism assumes that recoil radiation is soft. This large value is however relatively innocuous, because, as we will see, for the joint-resummed cross section the dominant $Q_{T}$ values are quite moderate, typically a few percent of the hard scale. The benefit of this large cut-off value is that, while recoil effects are still dominated by soft gluons, it allows us to recover numerically the threshold-resummed result, in the manner described at the end of section 2. For bottom quark production we choose $\bar{\mu}=30 \mathrm{GeV}$, the motivation for this large value being similar to that for the top quark.

Before we show results, let us state our default choices for various input parameters. For these and other plots we use the NLO GRV parton density set of Ref. [22] corresponding to $\alpha_{s}\left(M_{Z}\right)=0.114$, with the evolution code of Ref. 23], changing flavor number at $\mu=m_{c}(1.4 \mathrm{GeV}), m_{b}(4.5 \mathrm{GeV})$. We chose the factorization and renormalization scale equal to the transverse mass $m_{T}$. For the non-perturbative Gaussian smearing parameter $g$ in Eq. (14) we took $g=1 \mathrm{GeV}^{2}$. Furthermore, to avoid spurious singular behavior at the subleading level, we used, following [3]

$$
\chi(b Q, N)=\bar{b}+\frac{\bar{N}}{1+\eta \bar{b} / \bar{N}}, \quad \bar{b}=\frac{b Q e^{\gamma_{E}}}{2},
$$

with $\eta=1 / 4$.

We begin with top quark $(m=178 \mathrm{GeV})$ production, and compare in Figure 1 jointresummed, threshold-resummed and the exact LO and NLO calculations for the top quark $p_{T}$ spectrum produced in the $q \bar{q}$ channel at the Tevatron $(\sqrt{S}=1.96 \mathrm{TeV})$. We observe
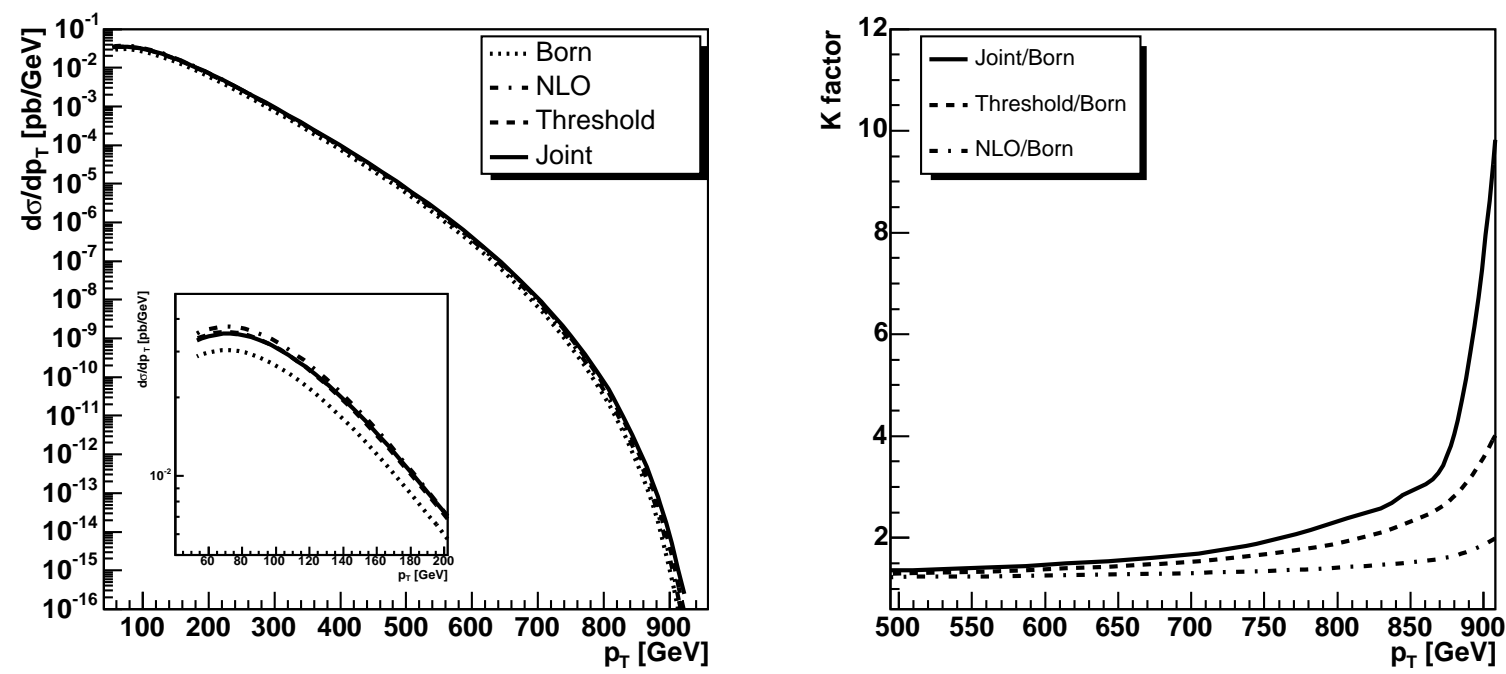

Figure 1: Top quark $p_{T}$ spectra and $K$-factors for the $q \bar{q}$ channel.

that, while the resummed and NLO curves are close for small and moderate $p_{T}$ (the inset provides a somewhat better view of the low $p_{T}$ region), for large $p_{T}$ values the resummed curves depart significantly from the NLO curve. Of course, cross sections for 
top quark production at such large $p_{T}$ at the Tevatron are far too small to be measured, so that our plots at large $p_{T}$ have only theoretical interest. For such large $p_{T}$ values, the hadronic threshold, defined in Eq. (4), approaches the partonic one, where larger $N$ values dominate, a prerequisite for seeing significant effects for both resummations. The enhancements relative to the Born cross section are shown in the form of a K-factor ${ }^{2}$ in Figure 1. As expected, threshold resummation produces an overall enhancement of the cross section that increases with increasing $p_{T}$, yielding e.g. a $35 \%$ enhancement over NLO at $p_{T}=800 \mathrm{GeV}$. Joint resummation almost doubles that effect, which can be understood from the arguments given in the introduction: the joint-resummed enhancement at large $p_{T}$ effectively constitutes a smearing of the threshold-resummed $p_{T}$ spectrum by a resummed recoil function.
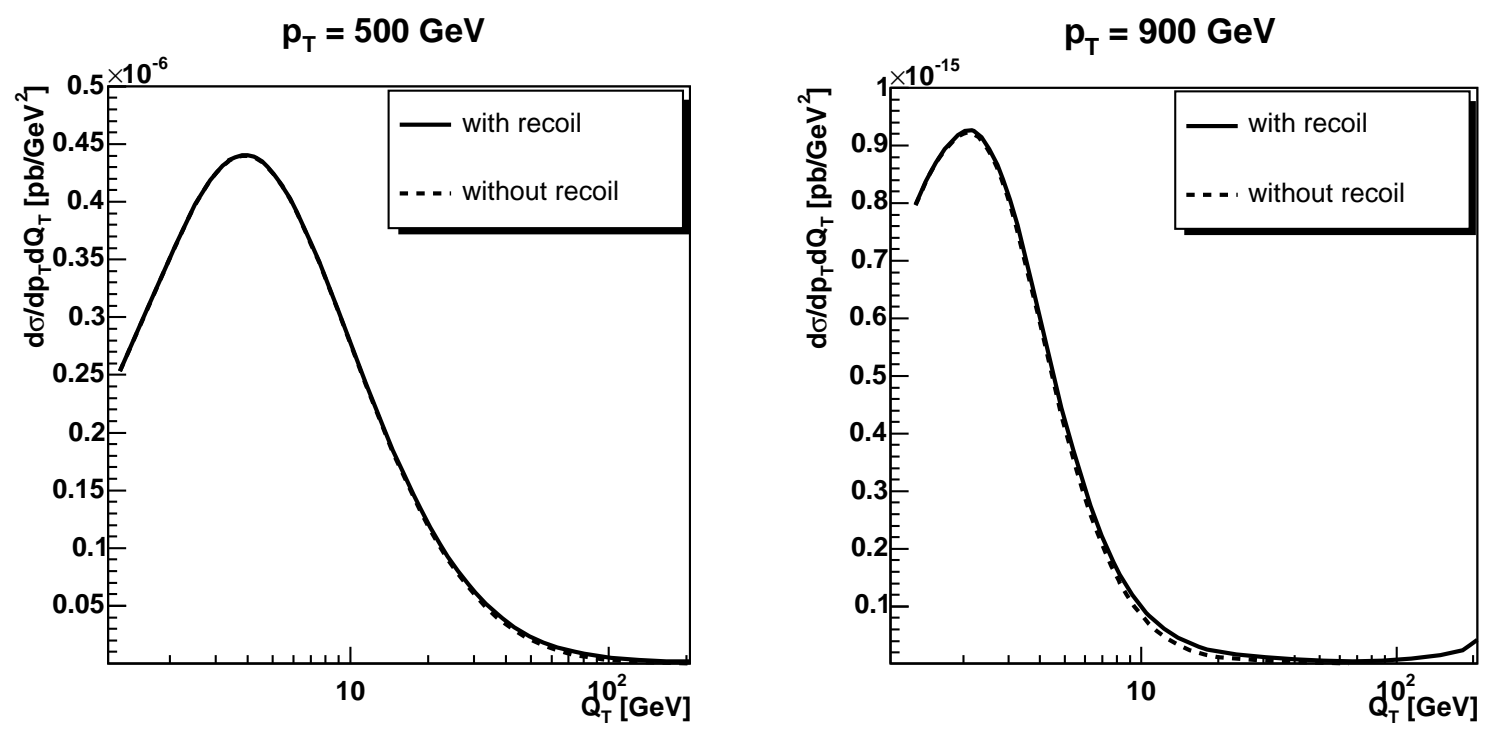

Figure 2: Top quark profile functions (Eq. (13)) for the $q \bar{q}$ channel, at $p_{T}=500$ and 900 $\mathrm{GeV}$.

Before showing results for the $g g$ channel, we exhibit in Fig. 2 the $Q_{T}$ profile of Eq. (13) for two rather large $p_{T}$ values, in analogy to Fig. 1 in Ref. [5]. In agreement with Fig. 1] we observe a small enhancement over the threshold-resummed result, in particular at very large $p_{T}$. The peak of the distribution is at relatively soft values of $Q_{T}$, a few percent of the hard scale $m_{T}$. For $p_{T}=500 \mathrm{GeV} Q_{T}$ reaches $\bar{\mu}$ before the bound in Eq. (20), so that there is no divergence for larger $Q_{T}$. For $p_{T}=900 \mathrm{GeV}$ there is a region of $Q_{T}$ phase space where the bound in Eq. (20) is saturated and the cross section diverges. The onset of that singularity is visible in Fig. 2, but because of our choice $\bar{\mu}=200 \mathrm{GeV}$ it does contribute a small amount to the $K$-factor in Fig. 1 at the largest $p_{T}$ value. We also observe that, in case of top production, for $p_{T} \lesssim 863.7 \mathrm{GeV}$, the disk $D_{2}$ in eq. (21) is fully contained in the disk $D_{1}$ which represents the bound in Eq. (20). Therefore below that value, which includes all $p_{T}$ values accessible experimentally, all the enhancement of the joint resummed cross section with respect with the threshold resummed is to be ascribed to recoil effects.

In Figs. 3 we show results analogous to Fig. 1, now for the $g g$ channel summed over the two color states, while in Fig. 4 we show the K-factors per color state. The K-factors show

\footnotetext{
${ }^{2}$ Normalized to the Born cross section.
} 

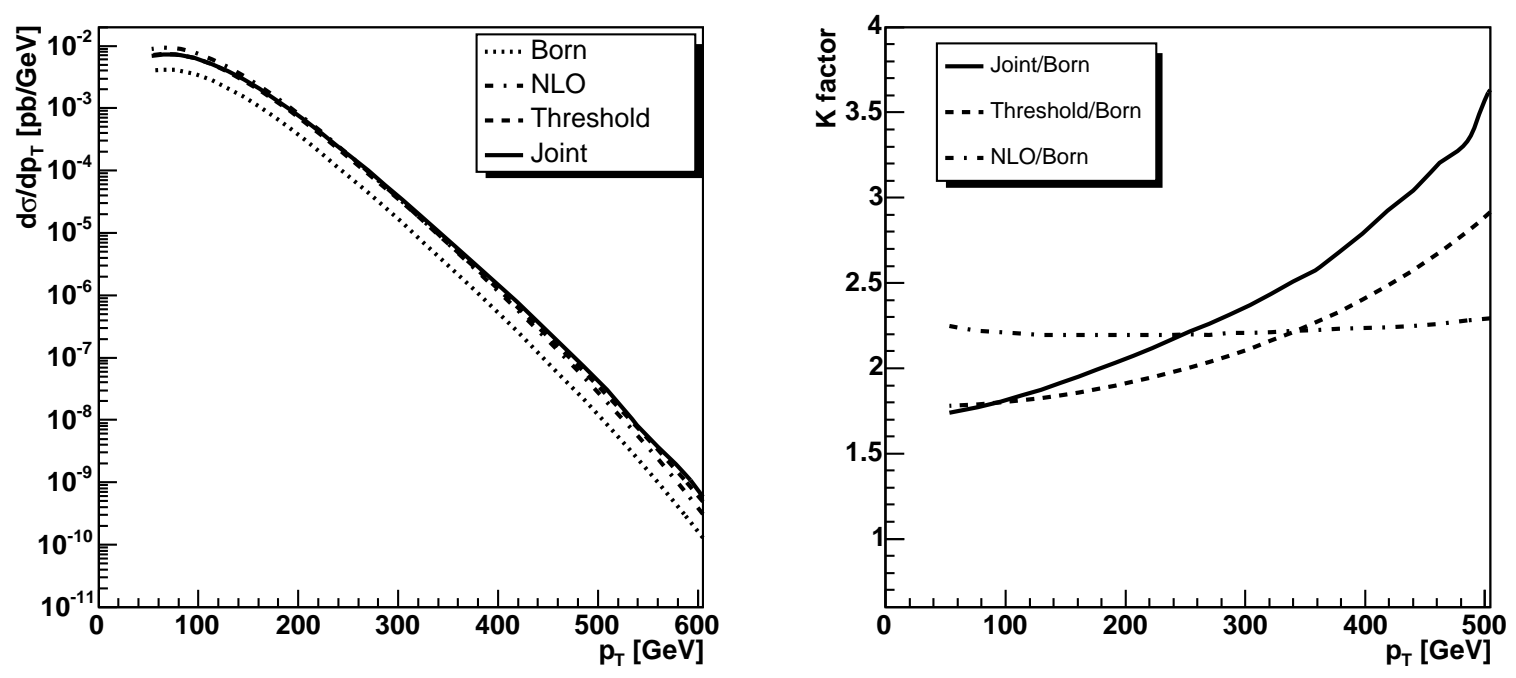

Figure 3: Top quark $p_{T}$ spectra and corresponding $K$-factors for the sum of color-singlet and color-octet $g g$ channels.
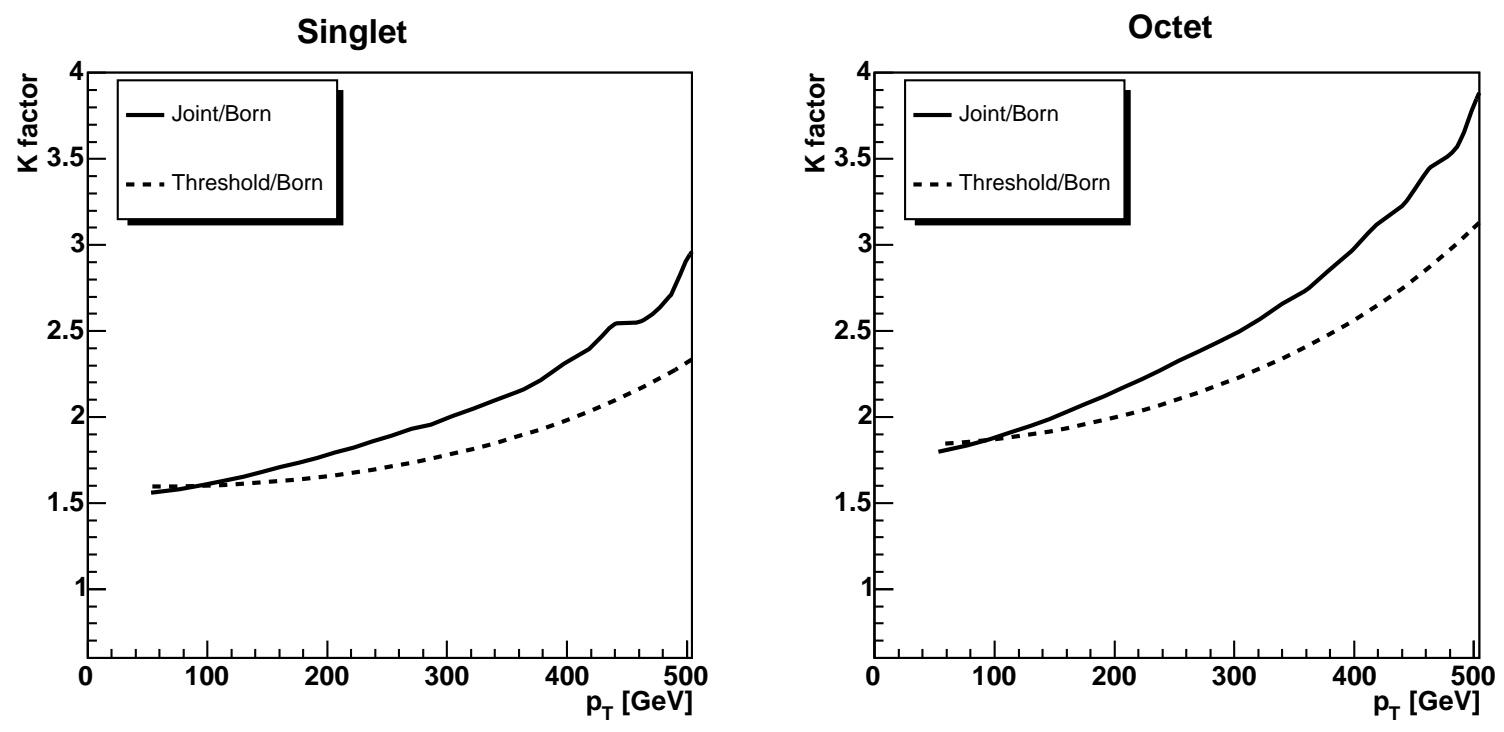

Figure 4: $K$-factors for the incoming color-singlet and color-octet $g g$ channels. 
a significant enhancement for joint resummation with respect to threshold resummation, increasing with increasing $p_{T}$, and strongest for octet production. The resummed spectra are harder than the fixed order ones. The increased enhancement of the $g g$ channel over the $q \bar{q}$ channel is due to the larger value for $A_{g}$ compared to $A_{q}$ in Eq. (14).

We have verified that both resummed cross sections have a reduced scale dependence with respect to $\mathrm{LO}$, as expected on general grounds [24, 25]. The reduction in the resummed cross section is somewhat less than in the exact NLO cross section, suggesting that a matched NLL-NLO calculation would do better still in this regard. Doing such a calculation is however beyond the scope of our paper.

In order to assess the relevance of the last term on the right hand side in Eq. (11), which encodes the differences between contributions from octet and singlet soft anomalous dimensions, we computed the $p_{T}$ spectrum for the $q \bar{q}$ channel with and without this term. We found an effect ranging from $8 \%$ enhancement with this term included at lower $p_{T}$ to about $10 \%$ at higher $p_{T}$. For the octet $g g$ channel we found the enhancement over the singlet-only to be 12 to $15 \%$.

Turning now to the bottom quark $p_{T}$ spectrum, we took as kinematic conditions, for illustrative purposes, $p p$ collisions at the HERA-B cm energy $(41.6 \mathrm{GeV})$. In these settings the gluon channel is more dominant, and effects of joint resummation are more noticeable than for top production in the above. Fig. 5 is analogous to Fig. 10 but the effect of joint resummation are stronger now, which holds as well for the $g g$ channel in Fig. 6. In Fig. 5 ,
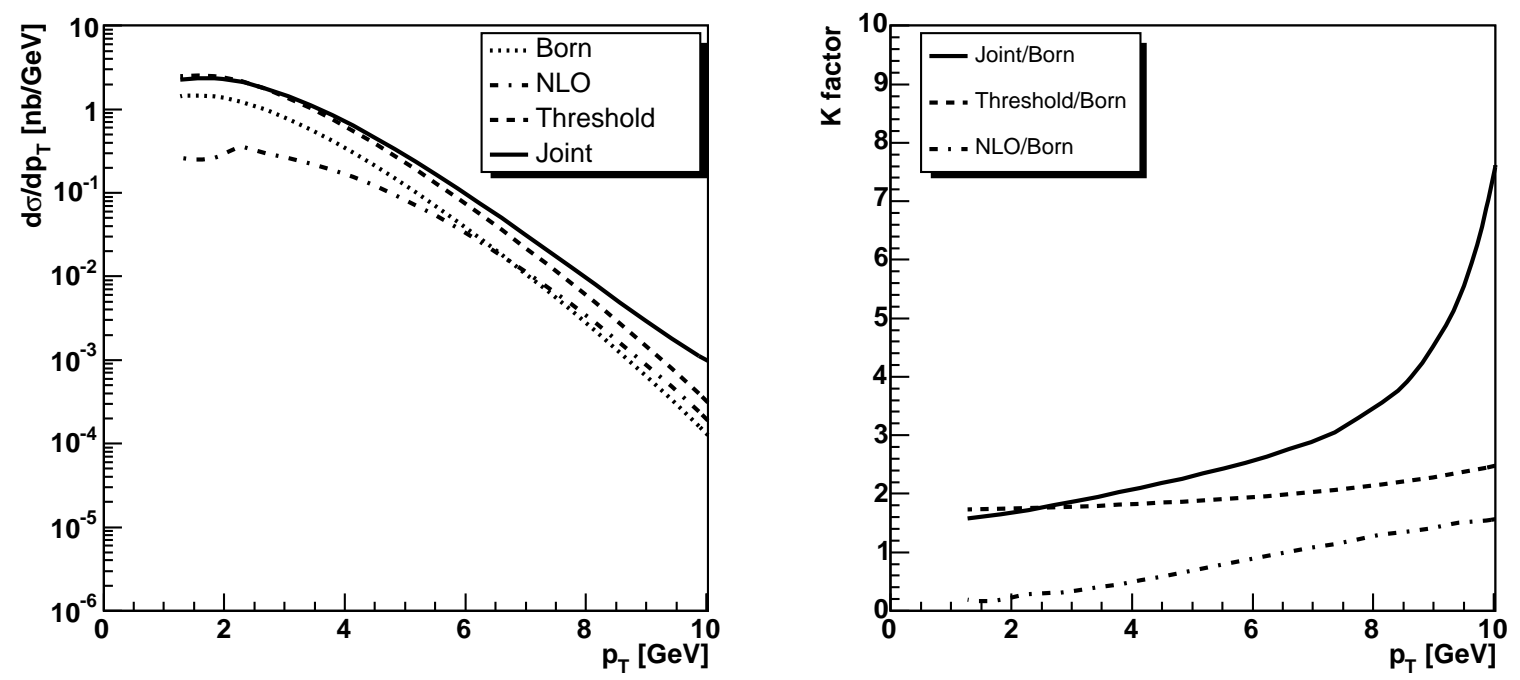

Figure 5: Bottom quark $p_{T}$ spectra and $K$-factors for the $q \bar{q}$ channel.

for the $q \bar{q}$ channel a significant effect at lower $p_{T}$ of virtual contributions in the NLO cross sections, not present in the resummed versions is visible, which is much less in the $g g$ channel. The $K$-factors are large (which is not unusual [7, 14, 17] for this kinematical configuration), with a clear distinction between threshold and joint resummation. The $Q_{T}$ profile of the enhancement at two values of $p_{T}$ is shown in Fig. 7 . Here is there no divergence, because the bound Eq. (201) is simply not reached. 

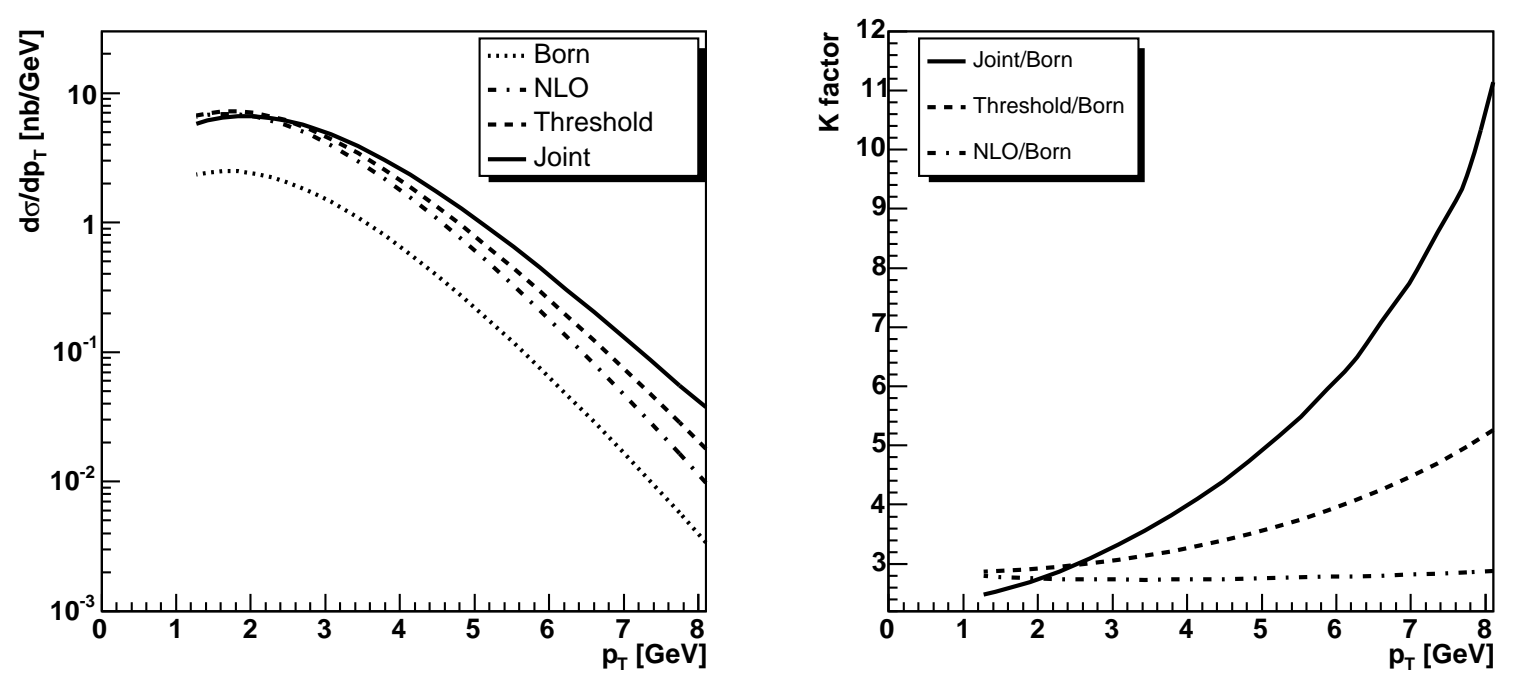

Figure 6: Bottom quark $p_{T}$ spectra and $K$-factors for the $g g$ channel.
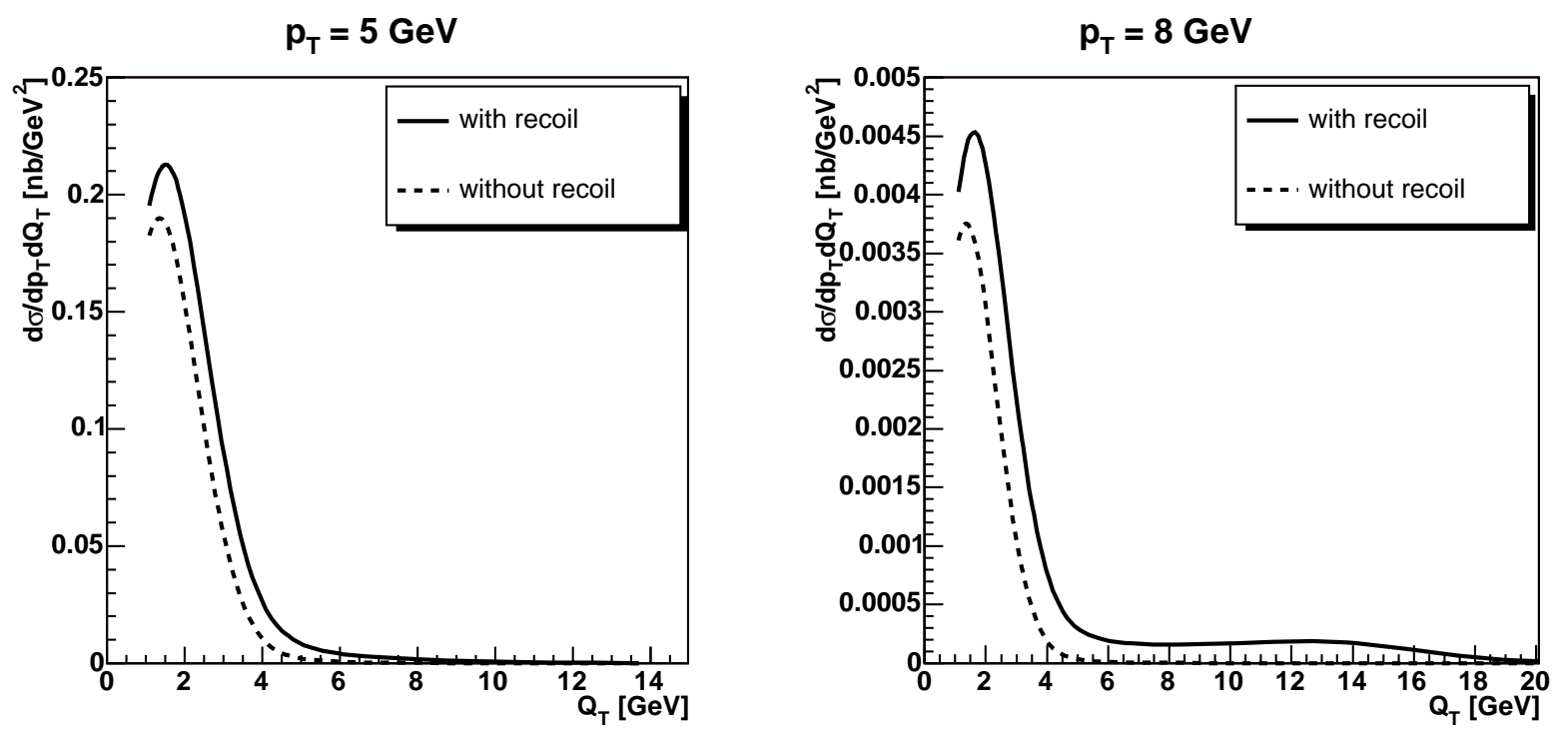

Figure 7: Bottom quark profile functions (Eq. (13) ) for the $g g$ channel, at $p_{T}=5$ and 8 $\mathrm{GeV}$. 


\section{Conclusions}

In this paper we derived the joint threshold and recoil resummed heavy quark transverse momentum distributions, to NLL accuracy. As a corollary we obtained the pure threshold resummed result as well. The resummed distributions contain two mutually incoherent components, associated with different color states (singlet and octet) of the heavy quark pair. These components differ by their underlying Born process and by anomalous dimensions governing the pattern of wide-angle soft emission. We provided a probabilistic interpretation of these anomalous dimensions.

For top quark production at the Tevatron, and bottom quark production at HERA-B, we studied the dependence of these results on the color state, and the difference with threshold-resummation. We found that joint resummation gives results noticeably different from threshold resummation, and that for a realistic range of transverse momentum the kinematic singularity present in the formalism, which is very visible in applications to massless (e.g. prompt photon) particle $p_{T}$ spectra, is well-screened by the heavy quark mass. Not surprisingly, resummation effects are larger in the $g g$ channel than the $q \bar{q}$ channel, and more noticeable for bottom-quark spectra at HERA-B than top-quark spectra at the Tevatron. The effect of the pure octet part of the anomalous dimension is noticeable. We hope that this study, taken together with recent other ones [3, 4, 6] contributes to developing joint resummation as a viable framework for making QCD predictions.

\section{Acknowledgments}

This work was supported by the Foundation for Fundamental Research of Matter (FOM) and the National Organization for Scientific Research (NWO). We are also grateful to Andreas Vogt for support in the use of the evolution code of Ref. [23].

\section{References}

[1] H.-n. Li, Phys. Lett. B454, 328 (1999), hep-ph/9812363.

[2] E. Laenen, G. Sterman, and W. Vogelsang, Phys. Rev. D63, 114018 (2001), hep-ph/0010080.

[3] A. Kulesza, G. Sterman, and W. Vogelsang, Phys. Rev. D66, 014011 (2002), hep-ph/0202251.

[4] A. Kulesza, G. Sterman, and W. Vogelsang, Phys. Rev. D69, 014012 (2004), hep-ph/0309264.

[5] E. Laenen, G. Sterman, and W. Vogelsang, Phys. Rev. Lett. 84, 4296 (2000), hep-ph/0002078.

[6] G. Sterman and W. Vogelsang, (2004), hep-ph/0409234.

[7] N. Kidonakis and R. Vogt, Eur. Phys. J. C36, 201 (2004), hep-ph/0401056.

[8] N. Kidonakis and R. Vogt, Eur. Phys. J. C33, S466 (2004), hep-ph/0309045.

[9] P. Nason, S. Dawson, and R. K. Ellis, Nucl. Phys. B327, 49 (1989). 
[10] W. Beenakker, H. Kuijf, W. L. van Neerven, and J. Smith, Phys. Rev. D40, 54 (1989).

[11] W. Beenakker, W. L. van Neerven, R. Meng, G. A. Schuler, and J. Smith, Nucl. Phys. B351, 507 (1991).

[12] M. L. Mangano, P. Nason, and G. Ridolfi, Nucl. Phys. B373, 295 (1992).

[13] J. C. Collins, D. E. Soper, and G. Sterman, in Perturbative Quantum Chromodynamics, A.H. Mueller ed., World Scientific, Singapore, 1989.

[14] R. Bonciani, S. Catani, M. L. Mangano, and P. Nason, Nucl. Phys. B529, 424 (1998), hep-ph/9801375.

[15] N. Kidonakis and G. Sterman, Phys. Lett. B387, 867 (1996).

[16] N. Kidonakis and G. Sterman, Nucl. Phys. B505, 321 (1997), hep-ph/9705234.

[17] N. Kidonakis, E. Laenen, S. Moch, and R. Vogt, Phys. Rev. D64, 114001 (2001), hep-ph/0105041.

[18] N. Kidonakis, G. Oderda, and G. Sterman, Nucl. Phys. B531, 365 (1998), hep-ph/9803241.

[19] N. Kidonakis, G. Oderda, and G. Sterman, Nucl. Phys. B525, 299 (1998), hep-ph/9801268.

[20] M. Cacciari, M. Greco, and P. Nason, JHEP 05, 007 (1998), hep-ph/9803400

[21] M. Cacciari and M. Greco, Nucl. Phys. B421, 530 (1994), hep-ph/9311260.

[22] M. Gluck, E. Reya, and A. Vogt, Eur. Phys. J. C5, 461 (1998), hep-ph/9806404.

[23] A. Vogt, (2004), hep-ph/0408244.

[24] G. Oderda, N. Kidonakis, and G. Sterman, (1999), hep-ph/9906338, in proceedings of Physics with a high luminosity polarized electron ion collider, Bloomington, Ind. 1999.

[25] G. Sterman and W. Vogelsang, in proceedings of Physics at Run II: QCD and Weak Boson Physics Workshop, edited by U. Baur, R.K. Ellis, D. Zeppenfeld. Batavia, Fermilab, 2000, hep-ph/0002132. 